\title{
Rotationally Symmetric Ferrofluid Flow and Heat Transfer in Porous Medium with Variable Viscosity and Viscous Dissipation
}

\author{
P. $\operatorname{Ram}^{\dagger}$ and V. Kumar \\ Department of Mathematics, NIT Kurukshetra, Haryana 136119, India \\ †Corresponding Author Email: parasram_nit@yahoo.co.in
}

(Received March 26, 2013; accepted May 27, 2013)

\begin{abstract}
The analysis of three dimensional rotationally symmetric boundary layer flow of field dependent viscous ferrofluid saturating porous medium is performed. The fluid under consideration is electrically nonconducting incompressible magnetic fluid. The flow is generated due to the rotation of an infinite disk maintained at a uniform temperature. The momentum equations give rise to nonlinear coupled boundary value problem which is solved using Finite Difference and Newton methods. The numerical solutions for the governing nonlinear differential equations are obtained over the entire range of physical parameters. The effects of field dependent viscosity, permeability parameter, Prandtl number $(P r)$ and Eckert number $(E c)$ on various flow characteristics are discussed in detail and presented graphically. A special attention has been paid to study the effects of viscous dissipation on thermal boundary layer. Appreciable effects of these physical parameters are recorded on boundary layer displacement thickness, skin friction coefficients and rate of heat transfer.
\end{abstract}

Keywords: Ferrofluid, Porous medium, Magnetic field, Viscous dissipation, Finite difference method.

$\begin{array}{llll} & & \text { NOMENCLATURE } & \\ \vec{B} & \text { magnetic induction } & R_{e} & \text { rotational Reynolds number, }\left(r^{2} \omega\right) / v \\ C_{p} & \text { specific heat at constant pressure } & T & \text { fluid temperature } \\ C_{f_{r}} & \text { radial skin friction coefficient } & T_{w} & \text { temperature at the wall } \\ C_{f_{t}} & \text { tangential skin friction coefficient } & T_{\infty} & \text { free stream temperature } \\ d & \text { boundary layer displacement thickness } & u & \text { radial velocity } \\ E & \text { dimensionless radial velocity } & v & \text { tangential velocity } \\ E c & \text { Eckert number, }\left(r^{2} \omega^{2}\right) /\left(\left(T_{w}-T_{\infty}\right) C_{p}\right) & w & \text { axial velocity } \\ F & \text { dimensionless tangential velocity } & \alpha & \text { dimensionless distance from the disk } \\ G & \text { dimensionless axial velocity } & \beta & \text { permeability parameter } v /\left(k_{0} \omega\right) \\ \vec{H} & \text { applied magnetic field } & \vec{\delta} & \text { coefficient of viscosity variation } \\ k & \text { thermal conductivity } & \eta & \text { reference fluid viscosity } \\ k_{0} & \text { porous permeability } & \eta_{m} & \text { magnetic field dependent viscosity } \\ \vec{M} & \text { magnetization } & \theta & \text { dimensionless temperature } \\ m & \text { MFD viscosity parameter } & \mu_{0} & \text { magnetic permeability in free space } \\ N u & \text { Nusselt number } & v & \text { reference kinematic viscosity } \\ P & \text { dimensionless fluid pressure } & \rho & \text { fluid density } \\ p & \text { reduced fluid pressure } & \tau_{r} & \text { radial shear stress } \\ P r & \text { Prandtl number, }\left(\eta C_{p}\right) / k & \tau_{t} & \text { tangential shear stress } \\ \vec{q} & \text { fluid velocity } & \omega & \text { angular velocity of disk }\end{array}$


First Author et al. / JAFM, Vol. x, No. x, pp. x-x, 200x.

\section{INTRODUCTION}

The ferrohydrodynamic flow and heat transfer over a rotating disk has been at center of research interests owing to the fact that disk driven FHD flows are not only of theoretical interests but are of practical significance in many areas, such as rotating machinery, thermal-power generating systems, computer storage devices, gas turbine rotors, medical equipments, air cleaning machines, crystal growth processes and aerodynamics. In turbine engines and other rotator type machine systems, the flow may be subjected to high operating temperatures and to ensure the optimal operation of machinery, it is vital to keep an accurate map of velocity and temperature fields. The flow of an ordinary viscous fluid near a rotating disk was first considered by Karman (1921) in one of the most influential articles ever written in aerodynamics, where the famous similarity transformations were introduced to reduce the governing Navier-Stokes equations (PDE's) into a set of coupled ordinary differential equations. That is why the flow near a rotating disk is widely known as Von Karman flow. Cochran (1934) improved Karman`s solution and calculated more accurate values by numerical integration. Cochran's hydrodynamic problem was extended to the flow starting impulsively from rest with some improvement by Benton (1966). Later, Evans (1969) studied the effects of uniform suction at the surface of porous rotating disk. Parter and Rajagopal (1984) studied the special solutions of Navier-Stokes equations for the swirling fluid flow between two infinite parallel plates rotating with some constant angular velocities. The comprehensive review and detailed discussions of the rotating flow due to a single and two disk systems are given by Owen and Rogers (1989).

In view of wide applications of rotating disk in industrial and other technological fields, the problem of flow near a rotating disk has been extended to magneto as well as ferro hydrodynamics. Smart materials such as ferrofluids are not available in free state in nature but are to be synthesized. Rosensweig (1985) has given an authoritative introduction to research on magnetic fluids in his monograph. For last three decades, ferrofluids have been used commercially in numerous devices like sensors, densimeters, accelerometers, pressure transducers etc and in sealing of hard disc drives, rotating X-ray tubes, rotating shafts and rods. These are used as lubricants in seals, bearings and dampers and as heat controller in electric motors and hi-fi speaker systems without the need of change in their geometric shape (Hathway (1979); Raj and Moslowitz (1990); Berkovsky and Bastovoi (1996)). In addition, ferrofluids can be used to deliver certain drugs to specific area of body and also treatment of cancer by heating the tumor soaked in ferrofluid by the means of alternating external magnetic field as an application in the field of bio-medicine. Attia (2001) discussed the unsteady magneto-hydrodynamic (MHD) flow of an incompressible viscous electrically conducting fluid due to an infinite rotating disk in the presence of an external uniform magnetic field. Turkyilmazoglu (2011) obtained the exact solution for the flow of viscous MHD fluid over a rotating disk. Ram and Kumar (2012) studied the effects of temperature dependent viscosity on steady axisymmetric ferrofluid flow over a stationary disk. The effects of rotation on the ferrofluid due to a rotating disk were studied by Ram and Sharma (2012).

In study of magnetic fluid flows, physical properties like fluid viscosity changes significantly with the applied magnetic field. So consideration of field dependent variable viscosity gives a realistic view of the flow field. In some research studies, the effects of magnetic field dependent (MFD) viscosity on electrically conducting and nonconducting fluid flows over a rotating disk have been considered. However, general information on magneto viscous effects in ferrofluid has been given in a monograph by Odenbach (2002). Sunil and Mahajan (2009) studied the nonlinear stability analysis of magnetized ferrofluid heated and soluted from below with MFD viscosity via generalized energy method by taking stress free boundaries. Ram et al. (2010) solved the non-linear differential equations under Neuringer - Rosensweig model for ferrofluid flow and discussed the effect of MFD viscosity on flow profiles. Nanjundappa et al. (2010) studied Benard-Marangoni ferroconvection in a ferrofluid layer in the presence of a uniform vertical magnetic field with MFD viscosity. Further, the effects of porosity and MFD viscosity on velocity and pressure profiles on a revolving ferrofluid over a rotating disk has been discussed by Ram and Sharma (2011). Veerraju et al. (2012) studied the variable viscosity effects on mixed convection boundary layer flow over a vertical plate in porous medium. The influence of MFD viscosity on ferrofluid flow in porous medium over a rotating disk is studied by Ram and Kumar (2012). The study of heat transfer in boundary layer flows is of great significance in various engineering applications such as drag reduction, transpiration, the design of thrust bearings and radial diffusers etc. Rahman et al. (2009) examined the heat transfer characteristics of a two dimensional flow of micro polar fluid passed a non-linear stretching sheet with temperature dependent variable viscosity. Chen (2010) studied the effects of joule heating and viscous dissipation on radiative heat transfer in MHD flow past a stretching surface. Sibanda and Makinde (2010) analyzed the effects of ohmic heating and viscous dissipation on axisymmetric MHD flow and heat transfer in porous medium. The MHD nonlinear flow and heat transfer past a stretching porous surface in porous medium is examined by Devi and Ganga (2010). The study of nanofluid flow with heat transfer in the presence of a porous rotating disk has been carried out by Bachok et al. (2011). Siddiqui et al. (2011) investigated the effects of partial slip on velocity profiles, temperature distribution and heat transfer in the fluid flow over a porous rotating disk. The disk driven steady flow and heat transfer of the 
power-law fluid is examined by Ming et al. (2011). Rashidi et al. (2012) employed the Homotopy Analysis method to obtain the analytical approximate solutions of fluid flow in porous medium and heat transfer. The flow and heat transfer characteristics of Oberbeck convection of chiral fluid in the presence of transverse magnetic field and viscous dissipation is studied by Rudraiah et al. (2013).

The objective of this paper is to study the effects of magnetic field dependent viscosity and viscous dissipation on heat transfer in steady axi-symmetric FHD boundary layer flow of an incompressible ferrofluid in porous medium. The equations governing the boundary layer flow in component form are non-dimensionalized using the similarity transformations. The resultant non-linear coupled differential equations are solved by subsequent use of the Finite Difference and Newton's method. Numerical calculations have been carried out in MATLAB and Flex PDE solver.

\section{MATHEMATICAL FORMULATION AND NUMERICAL SOLUTION}

\subsection{Mathematical Formulation}

Let us consider steady axially symmetric flow of an incompressible viscous electrically non-conducting ferrofluid with heat transfer in the presence of a rotating disk in porous medium with viscous dissipation, assuming that magnetic fluid is infinite in extent in positive z-direction. Electrically nonconducting disk is placed at $\mathrm{z}=0$ and rotates with some uniform angular velocity $\omega$ about $z$-axis. The flow is subjected to an externally applied magnetic field $\vec{H}$, and the magnetization $\vec{M}$ of fluid is aligned with the applied field. The disk is maintained at a uniform temperature $T_{w}$ and the free stream is kept at a constant temperature $T_{\infty}$.

The fluid viscosity is taken to be magnetic field dependent i.e. variable viscosity. Rosenswieg (1966) in his experimental work demonstrated that the magnetic field dependent viscosity has got exponential variation with respect to the magnetic field. However, for a small field variation, linear variation in MFD viscosity has been used, given as

$\eta_{m}=\eta(1+\vec{\delta} \cdot \vec{B})$

The differential equations governing the axisymmetric and steady boundary layer flow of ferrofluid are given as follows:

$$
\begin{aligned}
& \frac{\partial u}{\partial r}+\frac{u}{r}+\frac{\partial w}{\partial z}=0 \\
& u \frac{\partial u}{\partial r}-\frac{v^{2}}{r}+w \frac{\partial u}{\partial z}=-\frac{1}{\rho} \frac{\partial p}{\partial r}+\frac{\mu_{0}}{\rho}|\vec{M}| \frac{\partial}{\partial r}|\vec{H}| \\
& +\frac{\eta_{m}}{\rho}\left(\frac{\partial^{2} u}{\partial r^{2}}+\frac{\partial}{\partial r}\left(\frac{u}{r}\right)+\frac{\partial^{2} u}{\partial z^{2}}\right)-\frac{\eta_{m}}{\rho k_{0}} u
\end{aligned}
$$

$$
\begin{aligned}
& u \frac{\partial v}{\partial r}+\frac{u v}{r}+w \frac{\partial v}{\partial z}=\frac{\eta_{m}}{\rho}\left(\frac{\partial^{2} v}{\partial r^{2}}+\frac{\partial}{\partial r}\left(\frac{v}{r}\right)+\frac{\partial^{2} v}{\partial z^{2}}\right) \\
& -\frac{\eta_{m}}{\rho k_{0}} v \\
& u \frac{\partial w}{\partial r}+w \frac{\partial w}{\partial z}=-\frac{1}{\rho} \frac{\partial p}{\partial z}+\frac{\mu_{0}}{\rho}|\vec{M}| \frac{\partial}{\partial z}|\vec{H}| \\
& +\frac{\eta_{m}}{\rho}\left(\frac{\partial^{2} w}{\partial r^{2}}+\frac{1}{r} \frac{\partial w}{\partial r}+\frac{\partial^{2} w}{\partial z^{2}}\right)-\frac{\eta_{m}}{\rho k_{0}} w \\
& \rho C_{p}\left(u \frac{\partial T}{\partial r}+w \frac{\partial T}{\partial z}\right)=k\left(\frac{\partial^{2} T}{\partial r^{2}}+\frac{1}{r} \frac{\partial T}{\partial r}+\frac{\partial^{2} T}{\partial z^{2}}\right) \\
& +\eta_{m}\left[\left(\frac{\partial u}{\partial z}\right)^{2}+\left(\frac{\partial v}{\partial z}\right)^{2}\right]
\end{aligned}
$$

Boundary conditions for the flow are

$$
\begin{aligned}
& u=0 \quad v=r \omega, w=0 \text {, } \\
& p=0, T=T_{w} \text { at } z=0 \text {; } \\
& \left.u, v, p \rightarrow 0, T \rightarrow T_{\infty} \text { as } z \rightarrow \infty\right\}
\end{aligned}
$$

$w$ tends to some finite negative

value as $z \rightarrow \infty$

The second term on right hand side in the energy equation (6) is due to viscous dissipation. In the boundary layer flow of ferrofluid, the centrifugal force generated due to disk rotation is balanced by the radial pressure gradient and the radial component of magnetic force. So, the boundary layer approximation to Eq. (3) is

$$
-\frac{1}{\rho} \frac{\partial p}{\partial r}+\frac{\mu_{0}}{\rho}|\vec{M}| \frac{\partial}{\partial r}|\vec{H}|=-r \omega^{2}
$$

The similarity transformations suggested by Von Karman for rotating disk problem are as:

$$
u=r \omega E(\alpha), v=r \omega F(\alpha), w=\sqrt{v \omega} G(\alpha),
$$$$
p=\rho \omega v P(\alpha) \text { and } T-T_{\infty}=\left(T_{w}-T_{\infty}\right) \theta(\alpha)
$$

where $\alpha=\sqrt{\frac{\omega}{v}} z$.

On taking negligible $\mathrm{z}$-component of the magnetic field and using Eq. (9), the Eqs. (2 to 6) transform to a system of non-linear differential equations in non-dimensional variables $E, F, G, P$ and $\theta$ as

$$
\begin{aligned}
& G^{\prime}+2 E=0 \\
& m E^{\prime \prime}-G E^{\prime}-E^{2}+F^{2}-\beta m E-1=0 \\
& m F^{\prime \prime}-G F^{\prime}-2 E F-\beta m F=0 \\
& P^{\prime}-m G^{\prime \prime}+G G^{\prime}+\beta m G=0 \\
& \frac{1}{P r} \theta^{\prime \prime}-G \theta^{\prime}+m E c\left[\left(E^{\prime}\right)^{2}+\left(F^{\prime}\right)^{2}\right]=0
\end{aligned}
$$

The modified boundary conditions are 
P. Ram and V. Kumar / JAFM, Vol. 7, No. 2, pp. 357-366, 2014.

$E(0)=G(0)=P(0)=0, F(0)=\theta(0)=1$,

$E \rightarrow 0, F \rightarrow 0, P \rightarrow 0, \theta \rightarrow 0$ and

$G$ tends to some finite negative value

as $\alpha \rightarrow \infty$

Re-writing the differential Eqs. (11) to (14) by eliminating $E$ with the help of Eq. (10), we get

$$
\begin{aligned}
& 2 m G^{\prime \prime \prime}-2 G G^{\prime \prime}+\left(G^{\prime}\right)^{2}-4 F^{2} \\
& -2 \beta m G^{\prime}+4=0 \\
& m F^{\prime \prime}-G F^{\prime}+G^{\prime} F-\beta m F=0 \\
& P^{\prime}-m G^{\prime \prime}+G G^{\prime}+\beta m G=0 \\
& \frac{4}{P r} \theta^{\prime \prime}-4 G \theta^{\prime}+m E c\left[\left(G^{\prime \prime}\right)^{2}+4\left(F^{\prime}\right)^{2}\right]=0
\end{aligned}
$$

And the modified boundary conditions are

$$
\begin{aligned}
& G^{\prime}(0)=G(0)=P(0)=0, F(0)=\theta(0)=1, \\
& G^{\prime} \rightarrow 0, F \rightarrow 0, P \rightarrow 0, \theta \rightarrow 0 \text { and } \\
& G \text { tends to some finite negative value as } \\
& \alpha \rightarrow \infty
\end{aligned}
$$

\subsection{Numerical Solution}

The system of non-linear coupled differential Eqs. (16), (17) and (19) is solved with the boundary conditions (20) leaving the Eq. (18) as the dimensionless fluid pressure $P$ can directly be obtained from it, once the vertical component of velocity $G$ is made known. We adopted a second order numerical scheme, in which differential equations are discretized by approximating the involved derivatives by central differences, and subsequently, Newton's method is employed to solve the obtained nonlinear simultaneous difference equations. For numerical computations, the semi infinite integration domain $D^{*}=\{\alpha: 0 \leq \alpha<\infty\}$ is replace by the finite domain $D=\left\{\alpha: 0 \leq \alpha<\alpha_{\infty}\right\}$. It is to be noted that if this $\alpha_{\infty}$ is not chosen large enough, the numerical solution of the problem will not only depend upon the physical parameters but also on this $\alpha_{\infty}$. So, a sufficiently large value of $\alpha_{\infty}$ is taken so that the numerical solution closely approximate the asymptotic boundary conditions (20) and is not affected by imposing the terminal boundary conditions at a finite distance. The value $\alpha_{\infty}=12$ is found suitable to simulate $\alpha=\infty$ for all the cases shown in Figs. $1-10$.

The discretized equation corresponding to the reduced momentum Eqs. (16), (17) and the energy Eq. (19) are as follows:

$$
\begin{aligned}
& \frac{1}{h^{3}} m\left(G_{i+2}-2 G_{i+1}+2 G_{i-1}-G_{i-2}\right) \\
& -\frac{2}{h^{2}} G_{i}\left(G_{i+1}-2 G_{i}+G_{i-1}\right)+\frac{1}{4 h^{2}}\left(G_{i+1}-G_{i-1}\right)^{2} \\
& -4 F_{i}^{2}-\beta m \frac{1}{h}\left(G_{i+1}-G_{i-1}\right)+4=0
\end{aligned}
$$

$$
\begin{aligned}
& \frac{1}{h^{2}} m\left(F_{i+1}-2 F_{i}+F_{i-1}\right)-\frac{1}{2 h} G_{i}\left(F_{i+1}-F_{i-1}\right) \\
& +\frac{1}{2 h} F_{i}\left(G_{i+1}-G_{i-1}\right)-\beta m F_{i}=0 \\
& \frac{4}{h^{2} \operatorname{Pr}}\left(\theta_{i+1}-2 \theta_{i}+\theta_{i-1}\right)-\frac{2}{h} G_{i}\left(\theta_{i+1}-\theta_{i-1}\right) \\
& +m E c\left[\frac{1}{h^{4}}\left(G_{i+1}-2 G_{i}+G_{i-1}\right)^{2}\right] \\
& +m E c\left[\frac{1}{h^{2}}\left(F_{i+1}-F_{i-1}\right)^{2}\right]=0
\end{aligned}
$$

Now dividing the interval $(0,12)$ into

subintervals of length $h=\frac{1}{10}$ each, and $\alpha_{i}=\frac{i}{10}(0 \leq i \leq 120) \quad$ with $\quad \alpha_{0}=0 \quad$ and $\alpha_{120}=12$. Using the above value of $h$ in the Eqs. (21) to (23), we get

$$
\begin{aligned}
& 1000 m\left(G_{i+2}-2 G_{i+1}+2 G_{i-1}-G_{i-2}\right) \\
& -200 G_{i}\left(G_{i+1}-2 G_{i}+G_{i-1}\right)+25\left(G_{i+1}-G_{i-1}\right)^{2} \\
& -4 F_{i}^{2}-10 \beta m\left(G_{i+1}-G_{i-1}\right)+4=0 \\
& 100 m\left(F_{i+1}-2 F_{i}+F_{i-1}\right)-5 G_{i}\left(F_{i+1}-F_{i-1}\right) \\
& +5 F_{i}\left(G_{i+1}-G_{i-1}\right)-\beta m F_{i}=0 \\
& \frac{400}{\operatorname{Pr}}\left(\theta_{i+1}-2 \theta_{i}+\theta_{i-1}\right)-20 G_{i}\left(\theta_{i+1}-\theta_{i-1}\right) \\
& +m E c\left[10000\left(G_{i+1}-2 G_{i}+G_{i-1}\right)^{2}\right] \\
& +m E c\left[100\left(F_{i+1}-F_{i-1}\right)^{2}\right]=0
\end{aligned}
$$

And the further modified boundary conditions (20) are

$$
\begin{aligned}
& F_{0}=1, F_{12}=0, G_{0}=0, G_{-1}=G_{1}, \\
& G_{119}=G_{121}, \theta_{0}=1, \theta_{120}=0
\end{aligned}
$$

Now taking $i=1,2, \ldots, 119$ in the Eqs. (24) to (26), we get of three sets of non-linear simultaneous difference equations containing 119 equations each. From Eq. (24), we have 
P. Ram and V. Kumar / JAFM, Vol. 7, No. 2, pp. 357-366, 2014.

$$
\left.\begin{array}{l}
1000 m\left(G_{3}-2 G_{2}+2 G_{0}-G_{-1}\right) \\
-200 G_{1}\left(G_{2}-2 G_{1}+G_{0}\right)+25\left(G_{2}-G_{0}\right)^{2} \\
-4 F_{1}^{2}-10 \beta m\left(G_{2}-G_{0}\right)+4=0 \\
1000 m\left(G_{4}-2 G_{3}+2 G_{1}-G_{0}\right) \\
-200 G_{2}\left(G_{3}-2 G_{2}+G_{1}\right)+25\left(G_{3}-G_{1}\right)^{2} \\
-4 F_{2}^{2}-10 \beta m\left(G_{3}-G_{1}\right)+4=0 \\
\quad \vdots \\
1000 m\left(G_{121}-2 G_{120}+2 G_{118}-G_{117}\right) \\
-200 G_{119}\left(G_{120}-2 G_{119}+G_{118}\right) \\
+25\left(G_{120}-G_{118}\right)^{2}-4 F_{119}{ }^{2} \\
-10 \beta m\left(G_{120}-G_{118}\right)+4=0
\end{array}\right\}
$$

From Eq. (25), we have

$$
\left.\begin{array}{l}
100 m\left(F_{2}-2 F_{1}+F_{0}\right)-5 G_{1}\left(F_{2}-F_{0}\right) \\
+5 F_{1}\left(G_{2}-G_{0}\right)-\beta m F_{1}=0 \\
100 m\left(F_{3}-2 F_{2}+F_{1}\right)-5 G_{2}\left(F_{3}-F_{1}\right) \\
+5 F_{2}\left(G_{3}-G_{1}\right)-\beta m F_{2}=0 \\
\vdots \\
100 m\left(F_{120}-2 F_{119}+F_{118}\right) \\
-5 G_{119}\left(F_{120}-F_{118}\right)+5 F_{119}\left(G_{120}-G_{118}\right) \\
-\beta m F_{119}=0,
\end{array}\right\}
$$

From Eq. (26), we have

$$
\begin{aligned}
& \frac{400}{\operatorname{Pr}}\left(\theta_{2}-2 \theta_{1}+\theta_{0}\right)-20 G_{1}\left(\theta_{2}-\theta_{0}\right) \\
& +m E c\left[10000\left(G_{2}-2 G_{1}+G_{0}\right)^{2}\right] \\
& +m E c\left[100\left(F_{2}-F_{0}\right)^{2}\right]=0 \\
& \frac{400}{\operatorname{Pr}}\left(\theta_{3}-2 \theta_{2}+\theta_{1}\right)-20 G_{2}\left(\theta_{3}-\theta_{1}\right) \\
& +m E c\left[10000\left(G_{3}-2 G_{2}+G_{1}\right)^{2}\right] \\
& +m E c\left[100\left(F_{3}-F_{1}\right)^{2}\right]=0 \\
& \frac{400}{\operatorname{Pr}}\left(\theta_{120}-2 \theta_{119}+\theta_{118}\right) \\
& -20 G_{119}\left(\theta_{120}-\theta_{118}\right) \\
& +m E c\left[10000\left(G_{120}-2 G_{119}+G_{118}\right)^{2}\right] \\
& +m E c\left[100\left(F_{120}-F_{118}\right)^{2}\right]=0
\end{aligned}
$$

$$
\begin{aligned}
& G_{1}{ }^{1}=G_{1}{ }^{0}+a_{1}, G_{2}{ }^{1}=G_{2}{ }^{0}+a_{2}, \\
& \cdots, G_{119}^{1}=G_{119}^{0}+a_{119} \\
& F_{1}^{1}=F_{1}^{0}+b_{1}, \quad F_{2}{ }^{1}=F_{2}{ }^{0}+b_{2}, \\
& \cdots, F_{119}^{1}=F_{119}^{0}+b_{119} \\
& \theta_{1}^{1}=\theta_{1}^{0}+c_{1}, \quad \theta_{2}{ }^{1}=\theta_{2}{ }^{0}+c_{2}, \\
& \cdots, \theta_{119}^{1}=\theta_{119}^{0}+c_{119}
\end{aligned}
$$

Here subscript ' 0 ' denotes the initial value and ' 1 ' denotes the improved values after first iteration is performed. $a_{i}, b_{i}, c_{i}(1 \leq i \leq 119)$ are the perturbations from actual values, which may be positive or negative. It may take few steps more to converge as due to its dependence on initial guess. If initial guess is closer to the actual values, then it converges rapidly and comparatively less number of iterations is required, otherwise solution may not converge.

Using Newton's method, for the perturbations (31), the Eqs. (28) to (30) are linearized as follows:

$$
\left.\begin{array}{l}
f_{i}\left(G_{1}, \ldots, G_{119} ; F_{1}, \ldots, F_{119} ; \theta_{1}, \ldots, \theta_{119}\right) \\
+\sum_{k=1}^{119}\left(a_{k} \frac{\partial f_{i}}{\partial G_{k}}+b_{k} \frac{\partial f_{i}}{\partial F_{k}}+c_{k} \frac{\partial f_{i}}{\partial \theta_{k}}\right)=0 \\
g_{i}\left(G_{1}, \ldots, G_{119} ; F_{1}, \ldots, F_{119} ; \theta_{1}, \ldots, \theta_{119}\right) \\
+\sum_{k=1}^{119}\left(a_{k} \frac{\partial g_{i}}{\partial G_{k}}+b_{k} \frac{\partial g_{i}}{\partial F_{k}}+c_{k} \frac{\partial g_{i}}{\partial \theta_{k}}\right)=0 \\
h_{i}\left(G_{1}, \ldots, G_{119} ; F_{1}, \ldots, F_{119} ; \theta_{1}, \ldots, \theta_{119}\right) \\
+\sum_{k=1}^{119}\left(a_{k} \frac{\partial h_{i}}{\partial G_{k}}+b_{k} \frac{\partial h_{i}}{\partial F_{k}}+c_{k} \frac{\partial h_{i}}{\partial \theta_{k}}\right)=0 \\
\text { where } 1 \leq i \leq 119
\end{array}\right\}
$$

In matrix form the system can be written as $A X=B$, where

$$
\begin{aligned}
& A=\left[\begin{array}{llll}
\frac{\partial f_{1}}{\partial G_{1}} & \frac{\partial f_{1}}{\partial G_{2}} & \ldots . & \frac{\partial f_{1}}{\partial \theta_{119}} \\
\vdots & & & \\
\frac{\partial h_{119}}{\partial G_{1}} & \frac{\partial h_{119}}{\partial G_{2}} & \cdots & \frac{\partial h_{119}}{\partial \theta_{119}}
\end{array}\right] \quad X=\left[\begin{array}{c}
a_{1} \\
a_{2} \\
\vdots \\
\vdots \\
c_{119}
\end{array}\right], \\
& B=\left[\begin{array}{c}
-f_{1}\left(G_{1}, \ldots, G_{119} ; F_{1}, \ldots, F_{119} ; \theta_{1}, \ldots, \theta_{119}\right) \\
-f_{2}\left(G_{1}, \ldots, G_{119} ; F_{1}, \ldots, F_{119} ; \theta_{1}, \ldots, \theta_{119}\right) \\
\vdots \\
\vdots \\
-h_{119}\left(G_{1}, \ldots, G_{119} ; F_{1}, \ldots, F_{119} ; \theta_{1}, \ldots, \theta_{119}\right)
\end{array}\right]
\end{aligned}
$$
(30), we get a system 357 nonlinear coupled equations in 357 variables, which is solved using Newton's method.

Define the Eqs. (28) to (30) as functions $f_{i}\left(G_{1}, \cdots, G_{119} ; F_{1}, \cdots, F_{119} ; \theta_{1}, \cdots, \theta_{119}\right)=0$,

$g_{i}\left(G_{1}, \cdots, G_{119} ; F_{1}, \cdots, F_{119} ; \theta_{1}, \cdots, \theta_{119}\right)=0$ and
Solution of this system (i.e. the values of $a_{i}, b_{i}, c_{i}(1 \leq i \leq 119)$ ) is obtained in MATLAB. However the initial guess is taken with the help of PDE solver. The values of the variables $F, G$ and $\theta$ tend to the solution of Eqs. (28) - (30) when 
perturbations tend to zero. This obtained solution gives us the velocity and temperature profiles within the generated boundary layer over the surface of the disk.

Further, viscous ferrofluid layer in contact with the disk sets up the stress which opposes the rotation of the disk. The radial and tangential components of the generated stress are given by the Newtonian formulae as follows:

$$
\begin{aligned}
& \tau_{r}=\left[\eta_{m}\left(\frac{\partial u}{\partial z}+\frac{\partial w}{\partial r}\right)\right]_{z=0}=\eta m \mathrm{R}_{e}{ }^{1 / 2} \omega E^{\prime}(0) \\
& \tau_{t}=\left[\eta_{m}\left(\frac{\partial v}{\partial z}+\frac{1}{r} \frac{\partial w}{\partial \theta}\right)\right]_{z=0}=\eta m R_{e}^{1 / 2} \omega F^{\prime}(0)
\end{aligned}
$$

So radial and tangential skin frictions are respectively given by

$R_{e}^{1 / 2} C_{f_{r}}=m E^{\prime}(0)$

$R_{e}^{1 / 2} C_{f_{t}}=m F^{\prime}(0)$

The skin frictions calculated using Eq. (33) and Eq. (34) are presented in Table 1.

\begin{tabular}{|c|c|c|c|}
\hline \multicolumn{4}{|c|}{ Radial Skin Friction } \\
\hline \multicolumn{4}{|c|}{ At $\beta=2, \operatorname{Pr}=1, E c=0.5$} \\
\hline$m=1.0$ & $m=1.1$ & $m=1.2$ & $\begin{array}{r}m= \\
1.3\end{array}$ \\
\hline 0.5399 & 0.5940 & 0.6478 & 0.7021 \\
\hline \multicolumn{4}{|c|}{ At $m=1.2, \operatorname{Pr}=1, E c=0.5$} \\
\hline$\beta=0$ & $\beta=1$ & $\beta=2$ & $\beta=3$ \\
\hline 0.6479 & 0.6483 & 0.6478 & 0.6480 \\
\hline \multicolumn{4}{|c|}{ Tangential Skin Friction } \\
\hline \multicolumn{4}{|c|}{ At $\beta=2, \operatorname{Pr}=1, E c=0.5$} \\
\hline$m=1.0$ & $m=1.1$ & $m=1.2$ & $\begin{array}{r}m= \\
1.3\end{array}$ \\
\hline 0.6200 & 0.6818 & 0.7442 & 0.8061 \\
\hline \multicolumn{4}{|c|}{ At $m=1.2, \operatorname{Pr}=1, E c=0.5$} \\
\hline$\beta=0$ & $\beta=1$ & $\beta=2$ & $\beta=3$ \\
\hline 0.7439 & 0.7438 & 0.7442 & 0.7440 \\
\hline
\end{tabular}

Table 1 Radial and tangential skin frictions

Table 2 Boundary layer displacement thickness

\begin{tabular}{|c|c|c|c|}
\hline \multicolumn{4}{|c|}{ At $\beta=2, \operatorname{Pr}=1, E c=0.5$} \\
\hline$m=1.0$ & $m=1.1$ & $m=1.2$ & $m=1.3$ \\
\hline 2.3863 & 2.6279 & 2.8689 & 3.1093 \\
\hline At $m=1.2, \operatorname{Pr}=1, E c=0.5$ \\
\hline$\beta=0$ & $\beta=1$ & $\beta=2$ & $\beta=3$ \\
\hline 1.2892 & 2.0789 & 2.8689 & 3.6586 \\
\hline
\end{tabular}

The boundary layer displacement thickness $(d)$ is calculated as

$d=\frac{1}{r \omega} \int_{0}^{\infty} v d z=\int_{0}^{\infty} F(\alpha) d \alpha$
Also, the rate of heat transfer from the surface of disk is evaluated using the Fourier law as follows:

$q=\left(k \frac{\partial T}{\partial z}\right)_{z=0}=-k\left(T_{w}-T_{\infty}\right) \sqrt{\frac{\omega}{v}} \theta^{\prime}(0)$

Therefore, the Nusselt number $(\mathrm{Nu})$ is given by

$R_{e}^{1 / 2} N u=-\theta^{\prime}(0)$

After above computations, the boundary layer displacement thickness and the rate of heat transfer are presented in Table 2 and Table 3, respectively.

\begin{tabular}{|c|c|c|c|}
\hline \multicolumn{4}{|c|}{ At $\beta=2, P r=1, E c=0.5$} \\
\hline$m=1.0$ & $m=1.1$ & $m=1.2$ & $m=1.3$ \\
\hline 0.3229 & 0.3501 & 0.3865 & 0.4358 \\
\hline \multicolumn{4}{|c|}{ At $m=1.2, \operatorname{Pr}=1, E c=0.5$} \\
\hline$\beta=0$ & $\beta=1$ & $\beta=2$ & $\beta=3$ \\
\hline 0.3879 & 0.3872 & 0.3865 & 0.3854 \\
\hline \multicolumn{4}{|c|}{ At $m=1.2, \beta=2, P r=1$} \\
\hline$E c=0$ & $E c=0.5$ & $E c=1.0$ & $E c=1.6$ \\
\hline 0.3904 & 0.3865 & 0.3742 & 0.3625 \\
\hline \multicolumn{4}{|c|}{ At $m=1.2, \beta=2, E c=0.5$} \\
\hline \multicolumn{2}{|c|}{$\operatorname{Pr}=1$} & \multicolumn{2}{|c|}{$\operatorname{Pr}=7$} \\
\hline \multicolumn{2}{|c|}{0.3865} & \multicolumn{2}{|c|}{0.5423} \\
\hline
\end{tabular}

Table 3 Rate of heat transfer

\section{RESUlTS DISCUSSION}

Using the numerical technique, outlined in previous section, velocity and temperature distributions for considered flow are computed for various values of viscosity parameter $(m)$, permeability parameter $(\beta)$, Prandtl number $(P r)$ and Eckert number $(E c)$ and are displayed graphically in following Figs. (1 to $10)$. For $\beta=0$, the problem reduces to the case of ferrofluid flow with field dependent viscosity without porous medium in the presence of rotating disk (Ram et al., 2010). Prior to carrying out the calculations for changing parameters, the numerical method adopted in the present investigation has been validated by comparing the results for the reduced case $(\beta=0)$ to those obtained by Ram et al. (2010), and found them in quite agreement.

Figure 1 represents the radial velocity profiles for various values of permeability parameter for fixed value of viscosity parameter $m=1.2$. The radial velocity increases near the wall and after reaching the maximum value in each case, it starts decreasing and finally tends to zero. Here, positive value of radial velocity indicates the radial outward flow near the disk. The centrifugal force, generated due to rotation of the disk, throws the fluid near the disk in outward direction. It is observed that radial velocity increases on increasing the permeability parameter and attains the highest peak value for $\beta=3$. However, this increase is comparatively smaller near the disk surface. Also, higher values of 
permeability parameter reduce the rate of convergence. Effects of viscosity parameter on the radial velocity for a fixed permeability parameter $\beta=2$ are given in Fig. 2. Like the permeability parameter, increasing viscosity parameter increases the radial velocity, but effects of later are more prominent. Also convergence rate gets slower for higher values of $m$ and $\beta$ both.

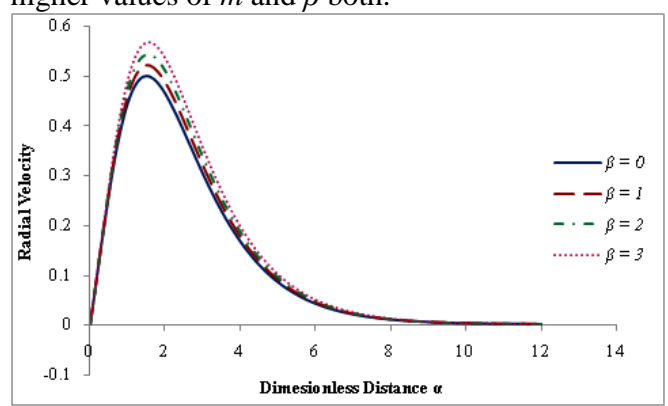

Fig.1. Radial velocity profiles for permeability parameter $\beta$ at $m=1.2, \operatorname{Pr}=1$ and $E c=0.5$

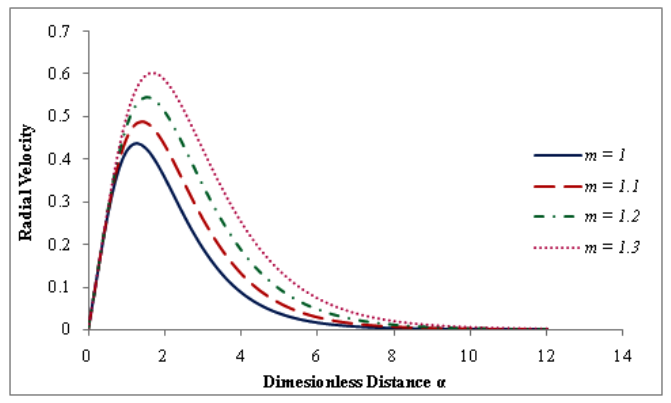

Fig. 2. Radial velocity profiles for viscosity parameter $m$ at $\beta=2, P r=1$ and $E c=0.5$

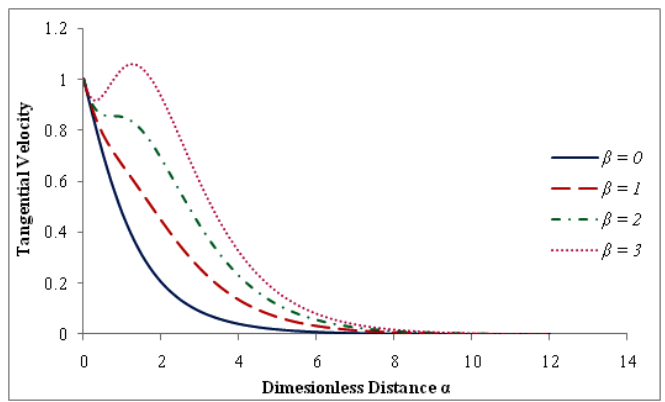

Fig. 3. Tangential velocity profiles for permeability parameter $\beta$ at $m=1.2, \operatorname{Pr}=1$ and $E c=0.5$

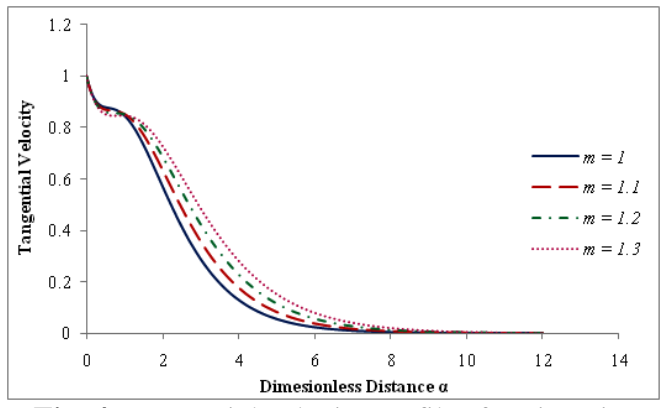

Fig. 4. Tangential velocity profiles for viscosity parameter $m$ at $\beta=2, \operatorname{Pr}=1 \& E c=0.5$

Effects of permeability parameter on tangential component of velocity for $m=1.2$ are illustrated in Fig. 3. And Fig. 4 shows the tangential velocity for change in viscosity parameter $m$ at a specified permeability parameter $\beta$. It is revealed that tangential velocity increases with increasing values of the parameter $\beta$ as well as with viscosity parameter $m$. It decreases smoothly from 1 to zero for $\beta=1 \& 2$, but a phenomenal change near the disk surface can be seen for greater values of $\beta$, where it once goes up for $\beta=3$. Tangential velocity takes the value 1 at the surface of the disk due to the no-slip condition at the wall, and then decreases to zero owing to the viscosity of the fluid. Further, late convergence of tangential velocity for higher values of both of the parameters is noticed.

Axial velocity profiles for different values of permeability parameter are depicted in Fig. 5 and for MFD viscosity parameter in Fig. 6. To compensate the radial outward flow of the ferrofluid on the surface of the disk, fluid flows axially downwards. So in both of the figures, the axial velocity remain negative. It is seen that axial velocity goes to more and more negative region as the field viscosity parameter increases, while gets affected negligibly due to change in the permeability parameter $\beta$.

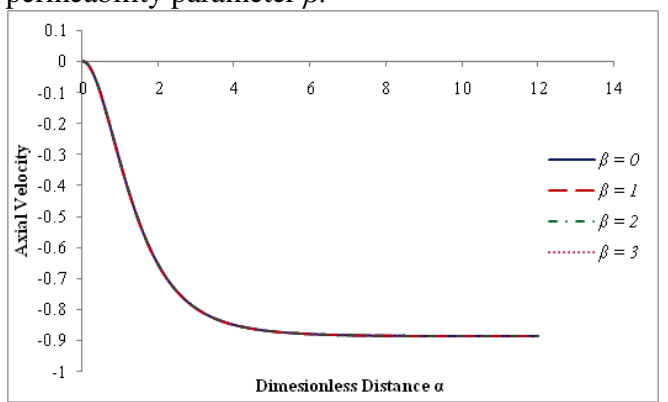

Fig. 5. Axial velocity profiles for permeability parameter $\beta$ at $m=1.2, P r=1$ and $E c=0.5$

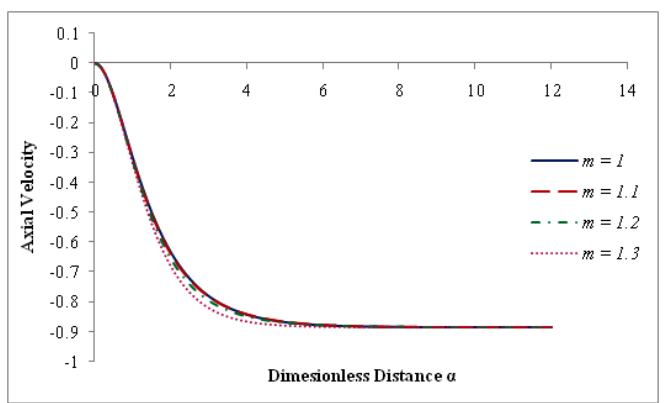

Fig. 6. Axial velocity profiles for viscosity parameter $m$ at $\beta=2, P r=1$ and $E c=0.5$

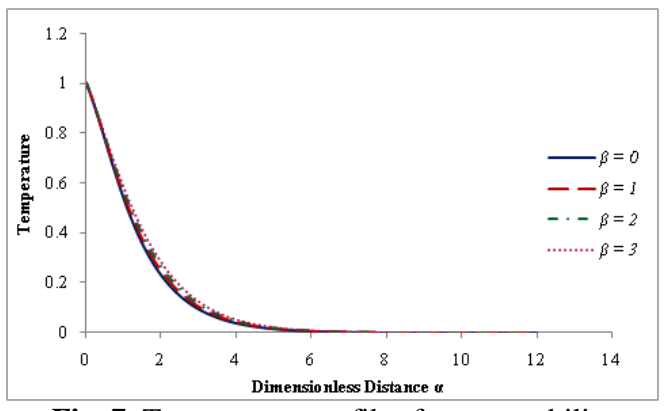

Fig. 7. Temperature profiles for permeability parameter $\beta$ at $m=1.2, P r=1$ and $E c=0.5$ 


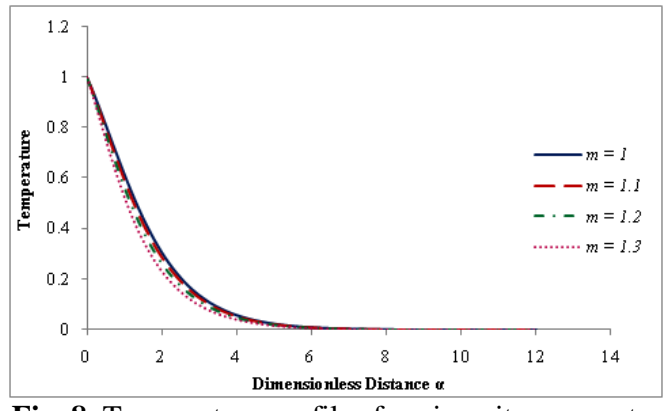

Fig. 8. Temperature profiles for viscosity parameter $m$ at $\beta=2, P r=1$ and $E c=0.5$

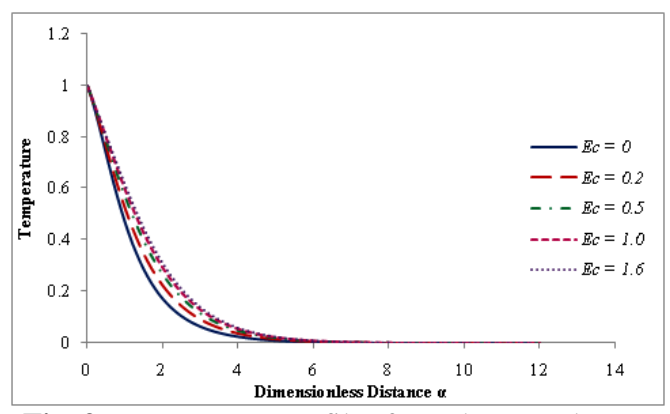

Fig. 9. Temperature profiles for Eckert number $E c$ at $m=1.2, \beta=2$ and $P r=1$

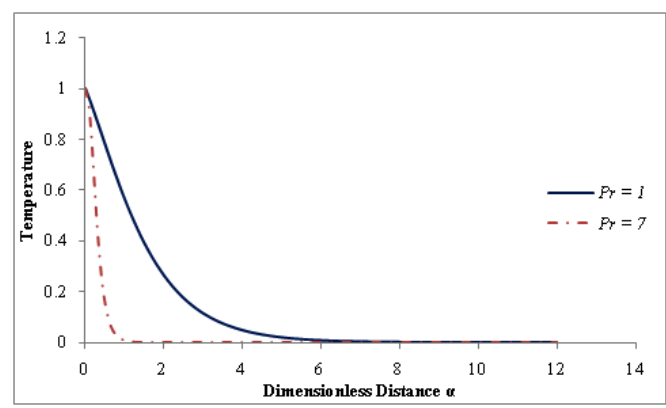

Fig. 10. Temperature profiles for the Prandtl number $\operatorname{Pr}$ at $m=1.2, \beta=2$ and $E c=0.5$

Figures 7 to 10 demonstrate the temperature distribution in the flow field for permeability parameter $(\beta)$, viscosity parameter $(m)$, Prandtl number $(P r)$ and Eckert number $(E c)$. It is observed that temperature increases on increasing the permeability parameter whereas it behaves conversely with respect to the viscosity parameter. However, the effects due to change in viscosity parameter are more prominent. From Fig. 9, it is clear that temperature increases on increasing the Eckert number i.e. boundary layer gets heated with increasing viscous dissipation. Whereas increasing value of the Prandtl number decreases the temperature distribution within the layer as demonstrated in Fig. 10. Moreover, the thermal boundary layer becomes thicker on increasing the permeability parameter as well as the Eckert number while becomes comparatively thinner with an increase in the viscosity parameter and the Prandtl number.

Impact of viscosity and permeability parameters on radial and tangential skin frictions are presented through the Table 1. It is noticed that radial as well as tangential skin frictions get increased on increasing the MFD viscosity parameter while the permeability parameter has negligible effects on these. Table 2 describes the effects of viscosity and permeability parameters on the boundary layer displacement thickness. Displacement thickness increases on increasing the both, the viscosity parameter and the permeability parameter, however, the effects of permeability parameter are more prominent. Further, behaviour of the numerical rate of heat transfer from the disk surface is shown in Table 3. It is depicted that rate of heat transfer increases with growing viscosity parameter and the Prandtl number, whereas, it behaves conversely for permeability parameter and the Eckert number.

\section{Conclusions}

The current research work draws light on the effects of MFD viscosity and viscous dissipation on the boundary layer flow of ferrofluid generated over a rotating disk in porous medium. The main results of this work are summarized as follows:

(i) Radial and tangential component of velocity and temperature get increased with growing permeability parameter $\beta$, while axial velocity remains almost same for it.

(ii) On increasing the MFD viscosity parameter $m$, radial and tangential velocities become large whereas axial velocity and temperature behave conversely.

(iii) Temperature distribution within the boundary layer decreases for higher Prandtl number whereas it increases with the Eckert number.

(iv) An increase in values of the $\beta \& E c$ thickens the thermal boundary layer, while for increasing $\operatorname{Pr}$ $\& m$, it becomes thinner.

(v) Radial and tangential skin frictions both become large on increasing the viscosity parameter whereas the permeability parameter has insignificant effects on it.

(vi) Increasing values of the permeability parameters thickens the generated boundary layer with insignificant effect of the viscosity parameter on it.

(vii) Numerical rate of heat transfer increases on increasing the viscosity parameter and the Pradtl number, whereas it behaves conversely for the permeability parameter and the Eckert number.

In nut shell, heat transfer in the ferrofluid flow over a heated rotating disk in porous medium is significantly affected by MFD viscosity, permeability of the medium and the viscous dissipation. And this flow can be controlled by proper tuning of these parameters depending upon its applications in various areas of science and engineering.

\section{REFERENCES}

Attia, H.A. (2001), "Transient flow of a conducting fluid with heat transfer due to an infinite rotating disk", International Communications in Heat and Mass Transfer, 28(3), 439-448. 
Bachok, N., Ishak, A. and Pop, I. (2011), "Flow and heat transfer over a rotating porous disk in a

nanofluid", Physica B, 406, 1767-1772.

Benton, E.R. (1966), "On the flow due to a rotating disk", Journal of Fluid Mechanics, 24(4), 781800 .

Berkovsky, B.M. and Bastovoi, V.G. (1996), Magnetic Fluids and Applications Handbook, Begall House Inc., New York.

Chen, C.H. (2010), "Combined effects of Joule heating and viscous dissipation on magnetohydrodynamic flow past a permeable, stretching surface with free convection and radiative heat transfer", Journal of Heat Transfer, 132(6).

Cochran, W.G. (1934), "The flow due to a rotating disk", Proceedings of Cambridge Philosophical Society, 30(3), 365-375.

Devi, S.P.A. and Ganga, B. (2010), "Dissipation effects on MHD nonlinear flow and heat transfer past a porous surface with prescribed heat flux", Journal of Applied Fluid Mechanics, 3(1), 1-6.

Evans, D.J. (1969), "The rotationally symmetric flow of a viscous fluid in the presence of an infinite rotating disk with uniform suction", Quarterly Journal of Mechanics and Applied Mathematics, XXII, 467-485.

Hathway, D.B. (1979), "Use of ferrofluid in moving coil loudspeakers", $d B$-Sound Engineering Magazine, 13(2), 42-44.

Karman, V. (1921), "Uber laminare und turbulente reibung”, Z. Angew. Math. Mech., 1(4), 233252.

Ming, C., Zheng, L. and Zhang, X. (2011), "Steady flow and heat transfer of the power law fluid over a rotating disk", International Communications in Heat and Mass Transfer, 38(3), 280-284.

Nanjundappa, C.E., Shivakumara, I.S. and Arunkumar, R. (2010), "Benard-Marangoni ferroconvection with magnetic field dependent viscosity", Journal of Magnetism and Magnetic Materials, 332(15), 2256-2263.

Odenbach, S. (2002), Magnetoviscous Effects in Ferrofluids, Springer-Verlag, Berlin.

Owen, J.M. and Rogers, R.H. (1989), Flow and Heat Transfer in Rotating Disk Systems, Research Stud. Press Ltd., John Wiley \& Sons.

Parter, S.V. and Rajagopal, K.R. (1984), "Swirling flow between rotating plates", Archive for Rational Mechanics and Analysis, 86(4), 305315.

Rahman, M.M., Rahman, M.A., Samad, M.A. and Alam, M.S. (2009), "Heat transfer in a micropolar fluid along a non-linear stretching sheet with a temperature-dependent viscosity and variable surface temperature",
International Journal of Thermophysics, 30(5), 1649-1670.

Raj, K. and Moskowitz, R. (1990), "Commercial applications of ferrofluids", Journal of Magnetism and Magnetic Materials, 85(1-5), 233-245.

Ram, P. and Kumar, V. (2012), "Effect of temperature dependent viscosity on revolving axi-symmetric ferrofluid flow with heat transfer", Applied Mathematics and Mechanics, 33(11), 1441-1452.

Ram, P. and Kumar, V. (2012), "Ferrofluid flow with magnetic field dependent viscosity due to rotating disk in porous medium", International Journal of Applied Mechanics, 4(4), 1250041.

Ram, P. and Sharma, K. (2011), "Revolving ferroluid flow under the influence of MFD viscosity and porosity with rotating disk", Journal of Electro Magnetic Analysis and Applications, 3(3), 378-386.

Ram, P. and Sharma, K. (2012), "On the revolving ferrofluid due to a rotating disk", International Journal of Nonlinear Science, 13(3), 317-324.

Ram, P. Bhandari, A. and Sharma, K. (2010), "Effect of magnetic field-dependent viscosity on revolving ferrofluid", Journal of Magnetism and Magnetic Materials, 322(21), 3476-3480.

Rashidi, MM., Pour, S.A.M., Hyat, T. and Obaidat, S. (2012), "Analytic approximate solutions for steady flow over a rotating disk in porous medium with heat transfer by homotopy analysis method", Computer and Fluids, 54, 19.

Rosensweig, R.E. (1966), "Buoyancy and stable levitation of a magnetic body immersed in a magnetizable fluid", Nature, 210, 613-614.

Rosensweig, R.E. (1985), Ferrohydrodynamics, Cambridge University Press, Cambridge.

Rudraiah, N., Sujatha, N. and Kumar, J.S. (2013), "Effects of variation of viscosity and viscous dissipation on Oberbeck magnetoconvection in a chiral fluid", Journal of Applied Fluid Mechanics, 6(2), 229-237.

Sibanda, P. and Mankide, O.D. (2010), "On steady MHD flow and heat transfer past a rotating disk in porous medium with ohmic heating and viscous dissipation", International Journal of Numerical Methods in Heat and Fluid Flow, 20(3), 269-285.

Siddiqui, A.M., Farooq, A.A., Ansari, A.R. and Haroon, T. (2011), "Exact solutions for laminar periodic non-axisymmetric slip flow of a viscous incompressible fluid due to a porous rotating disk", International Journal of Fluid Mechanics Research, 38(1), 71-84.

Sunil and Mahajan A. (2009), “A nonlinear stability analysis of a double diffusive magnetized ferrofluid with magnetic field dependent 
P. Ram and V. Kumar / JAFM, Vol. 7, No. 2, pp. 357-366, 2014.

viscosity", Journal of Magnetism and Magnetic Materials, 321(18), 2810-2820.

Turkyilmazoglu, M. (2011), "Exact solutions for the incompressible viscous magnetohydrodynamic fluid of a rotating disk flow", International Journal of Non-linear Mechanics, 46(1), 306-311.
Veeraju, N., Srinivasa Babu, K.S. and Rao, C.N.B. (2012), "Mixed convection at a vertical plate in a porous medium with magnetic field and variable viscosity", Journal of Applied Fluid Mechanics, Vol. 5 No. 4, 53-62. 